\title{
Crescimento e desenvolvimento de boto-cinza (Sotalia guianensis) do litoral do Espírito Santo
}

\author{
[Growth and development of estuarine dolphin (Sotalia guianensis) from \\ Espírito Santo coast] \\ A.P.M. Carvalho ${ }^{1}$, J. Ywasaki ${ }^{1}$, C.T. Azevedo ${ }^{2}$, A.S. Campos $^{3}$, F.F. Queiroz ${ }^{1}$, \\ L.A.E. Pontes ${ }^{1}$, L.A. Barbosa ${ }^{4}$, L.S. Silveira ${ }^{5}$ \\ ${ }^{1}$ Aluno de pós-graduação - Universidade Estadual do Norte Fluminense \\ Darcy Ribeiro - UENF - Campos dos Goytacazes, RJ \\ ${ }^{2}$ Aluna de graduação - UENF - Campos dos Goytacazes, RJ \\ ${ }^{3}$ Médica veterinária autônoma \\ ${ }^{4}$ Instituto de Conscientização Ambiental (Instituto ORCA) \\ ${ }^{5}$ Universidade Estadual do Norte Fluminense Darcy Ribeiro - Campos dos Goytacazes, RJ
}

\begin{abstract}
RESUMO
Estudou-se o desenvolvimento do boto-cinza (Sotalia guianensis) do litoral do Espírito Santo e verificouse se a análise das radiografias da aleta peitoral pode ser utilizada para tal. Trinta e sete botos passaram pela determinação do comprimento total (CT), análise do grau de fusionamento das vértebras e radiografia das aletas peitorais. O CT ao nascimento foi de $103,3 \mathrm{~cm}$ e o de animais maduros fisicamente $187,5 \mathrm{~cm}$. Observou-se correlação entre o CT, as epífises distais do rádio e da ulna e correlação entre o grau de fusionamento vertebral e a epífise distal do rádio. Concluiu-se que a análise radiográfica das aletas peitorais é uma boa maneira de avaliar o grau de maturação física do boto.
\end{abstract}

Palavras chave: Sotalia guianenis, radiografia, maturação física

\begin{abstract}
The aim of this study is to analyze the development of the estuarine dolphin from the coast of Espírito Santo and verify that the analysis of X-rays of pectoral fin can be used for such. Thirty-seven Sotalia guianensis were used, determining the TL, examination of the degree of fusion of the vertebrae and $X$-ray of pectoral fins. At birth the TL is $103.3 \mathrm{~cm}$ and in physically mature animals the TL is $187.5 \mathrm{~cm}$. The TL showed a significant correlation with the epiphyses of the distal radius and ulna and the degree of spinal fusion showed significant correlation with distal radius. It can be concluded that the X-ray analysis of the pectoral fins is a good way of assessing the physical maturation of these animals and can be used in routine procedures.
\end{abstract}

Keywords: Sotalia guianensis, radiography, physical maturity

\section{INTRODUÇÃO}

O boto Sotalia guianensis é um dos cetáceos mais encontrados no litoral brasileiro, sendo mais conhecido como boto-cinza (Sotalia guianensis). Habita regiões costeiras, (Flores, 2002; Nery et al; 2008) e é encontrado desde Honduras até o estado de Santa Catarina, no sul

Recebido em 5 de maio de 2011

Aceito em 1 de setembro de 2011

E-mail: annapcarvalho@yahoo.com.br do Brasil (Simões-Lopes, 1988; Flores, 2002; Fettuccia, 2006).

O boto é um dos menores cetáceos conhecidos; pertence à família Delphinidae (Fettuccia, 2006) e vive, em média, 30 anos. A maturidade sexual ocorre em média, aos seis anos de idade, e a maturidade física é alcançada aos sete anos com $185 \mathrm{~cm}$, segundo Ramos et al. (2000) em animais do litoral do Rio de Janeiro. 
O grau de desenvolvimento do indivíduo pode ser verificado pela análise dentária, pelo comprimento total, pela determinação da maturação sexual e pela análise da coluna vertebral (Moscatiello et al., 2008). O estudo radiográfico das nadadeiras peitorais para análise do desenvolvimento do esqueleto vem sendo realizado em diversas espécies de cetáceos, como Stenella coeruelalba, Tursiops truncatus, Delphinus delphis, Indopacetus pacificus e Balaenoptera edeni (Stockin et al., 2008), mas ainda não foi realizado em Sotalia guinanesis, especialmente os do litoral do Espírito do Santo. Em cetáceos, a aparência dos centros de ossificação secundária nas nadadeiras é avaliada e classificada de acordo com o padrão de Ogden et al. (1981).

O presente trabalho tem como objetivo analisar o desenvolvimento do boto-cinza e verificar se a análise das radiografias da aleta peitoral pode ser utilizada para tal.

\section{MATERIAL E MÉTODOS:}

Foram utilizados 37 odontocetos da espécie Sotalia guianensis, provenientes de encalhes ou captura acidental no litoral do estado do Espírito Santo. Todas as carcaças capturadas e identificadas encontram-se armazenadas no Instituto ORCA (Organização Consciência Ambiental). Foram coletados dados biológicos do comprimento total (CT), medido por meio de uma linha reta da ponta da parte superior da mandíbula até a comissura caudal. Não foram realizadas distinções sexuais.

As nadadeiras peitorais de cada indivíduo foram identificadas e radiografadas em pares em projeção laterolateral, utilizando-se uma régua escanográfica de $30 \mathrm{~cm}$. Os exames radiográficos foram realizados por aparelho comum, com a utilização de cassete digital AGFA $35 \times 43$, e a revelação foi feita por equipamento de radiografia digital AGFA. Foram realizados e avaliados os estágios de fusionamento do aspecto dos centros secundários de ossificação, visualizados do úmero até o metacarpo de acordo com Ogden et al. (1981). Segundo esses autores, estágio 0 é aquele em que ainda não houve formação do centro secundário de ossificação; no estágio 1, já aparece o centro de ossificação, mas este é menor que a latitude da epífise; no estágio 2 , o centro de ossificação está bem consolidado e faltam 50\% para completar a epífise; no estágio 3, há a formação de uma linha fisária radiolucente, com formação de osso mais denso entre a metáfise e o centro de ossificação; no estágio 4, há o fechamento da linha fisária com formação de trabéculas entre a metáfise e o centro de ossificação; no estágio 5, há o completo fusionamento da linha fisária com a formação de uma linha radiopaca; e no estágio 6 , começa o remodelamento ósseo com o desaparecimento da linha radiopaca.

Avaliou-se a maturidade física por meio da análise do grau de fusionamento das epífises dos corpos vertebrais. Foram considerados maduros os animais que apresentavam todas as epífises vertebrais fusionadas aos corpos vertebrais correspondentes (Perrin, 1975).

Foi utilizada a análise estatística descritiva para o comprimento total, em todos os animais, no grupo de animais menores e maiores que sete anos, no grupo de espécimes com vértebras sem início de fusionamento com as epífises, nas vértebras que apresentavam algumas epífises fusionadas e nas vértebras que se apresentavam completamente fusionadas. Foi utilizado o teste de correlação de Pearson (r) para estimar a correlação entre o grau de fusionamento das vértebras, o comprimento total e cada uma das epífises da nadadeira peitoral. Todas as análises foram realizadas pelo SAEG 9.1. (Sistema..., 2007).

\section{RESULTADOS E DISCUSSÃO}

Foram encontrados espécimes com idades entre zero e 20 anos. Foi associado zero ano de idade aos três espécimes $(8,1 \%)$ recém-nascidos encontrados, pois eles não apresentavam nem mesmo a linha neonatal na análise dentária. Neste caso, o comprimento total foi de 92, 96 e $122 \mathrm{~cm}$, média de $103,33 \mathrm{~cm}$. Este resultado é mais alto que o relatado por Schmielgeow (1990) em botos de litoral de São Paulo e Paraná, o qual descreveu que os animais neonatos apresentavam comprimento total entre 86,7 e $91,7 \mathrm{~cm}$. Rosas e Monteiro-Filho (2005), Ramos et al. (2000) e Santos et al. (2003) encontraram, ao nascimento, comprimento de $92,2 \mathrm{~cm}, 106 \mathrm{~cm}$ e $97,8 \mathrm{~cm}$, respectivamente.

Os menores de sete anos totalizavam 30 espécimes e em apenas 25 foi obtido o CT; os 
maiores que sete anos totalizavam sete espécimes. A média do CT nos menores de sete anos foi $154,77 \mathrm{~cm}$, e nos maiores de sete anos $187,5 \mathrm{~cm}$. Conforme relatado por Ramos et al. (2000), os animais maiores de sete anos são considerados maduros fisicamente.

Quanto ao grau de fusionamento das vértebras, 16 não apresentavam epífise vertebral fusionada, em 16 as epífises vertebrais estavam parcialmente fusionadas e em apenas cinco as epífises vertebrais estavam fusionadas. A média do CT para os animais sem nenhuma vértebra fusionada foi $143,2 \mathrm{~cm}$, para os animais com as vértebras parcialmente fusionadas, $168 \mathrm{~cm}$, e para os com as vértebras completamente fusionadas, $187,5 \mathrm{~cm}$.

Conforme relatado por Perrin (1975), os animais com todas as vértebras fusionadas são considerados maduros fisicamente. Como as médias dos dois grupos maduros fisicamente foi a mesma, sugere-se que os espécimes do Espírito Santo alcançam a maturidade física ao atingirem o CT de 187,5cm. Ramos et al. (2000), em animais da região norte do estado do Rio de Janeiro, relataram CT de $185,0 \mathrm{~cm}$ para os animais maduros fisicamente, e Schmielgeow (1990) estimou o CT dos animais maduros fisicamente do litoral de São Paulo em $180,1 \mathrm{~cm}$. Essa variação pode ter ocorrido porque os animais do litoral do Espírito Santo são considerados maiores do que os da região mais ao sul.

O CT é um parâmetro consagrado para analisar o grau de desenvolvimento dos animais (Ramos et al., 2000; Santos et al., 2003; Di Beneditto e Ramos, 2004). Os trabalhos mostram que há correlação entre CT e as análises das radiografias peitorais, especialmente as da epífise distal do úmero $(\mathrm{r}=0,7947)$, proximal do rádio $(\mathrm{r}=$ $0,7972)$ e proximal da ulna $(\mathrm{r}=0,7842)$, e distal do rádio $(\mathrm{r}=0,7822)$ e distal da ulna $(\mathrm{r}=$ 0,8077). Segundo Perrin (1975), há correlação entre o grau de fusionamento das epífises vertebrais e a epífise distal do rádio $(\mathrm{r}=0,7868)$. Todos esses resultados sugerem que as análises radiográficas podem ser utilizadas como mais uma forma de se avaliar a maturação física desses animais.

\section{CONCLUSÕES}

Os animais do litoral do Espírito Santo têm CT ao nascimento semelhante aos de outras regiões, mas estes atingem a maturidade física um pouco maiores. A análise da radiografia peitoral tem correlação significativa com o comprimento total e o grau de fusionamento das vértebras e, portanto, pode ser utilizada como método de avaliação de maturidade física destes animais.

\section{AGRADECIMENTOS}

À Coordenação de Aperfeiçoamento de Pessoal de Nível Superior (CAPES), pela concessão da bolsa, e ao Instituto ORCA e ao seu diretor, Lupércio Araújo Barbosa, por permitir a utilização do material que possibilitou este trabalho.

\section{REFERÊNCIAS}

DI BENEDITTO, A.P.M.; RAMOS R.M.A. Biology of the marine tucuxi dolphin (Sotalia fluviatilis) in south-eastern Brazil. J. Mar. Biol. Assoc., v.84, p.1245-1250, 2004.

FETTUCCIA, D.C. Comparação osteológica nas espécies do gênero Sotalia Gray, 1866 no Brasil (Cetacea, Delphinidae). $2006.107 \mathrm{f}$. Dissertação (Mestrado em Biologia Tropical e Recursos Naturais) - Instituto Nacional de Pesquisas da Amazônia, Universidade Federal do Amazonas, Manaus, AM.

FLORES, P.A.C. Tucuxi Sotalia fluviatilis In: PERRIN, W.F.; WÜRSIG, B.; THEWISSEN, G.M. Encyclopedia of marine mammals. San Diego: Academic, 2002. p.1267-1269.

MOSCATIELLO, V.A.M.; LEDERMAN, H.; MOSCATIELLO, R.A. et al. Maturação das vértebras cervicais e sua correlação com a idade óssea da mão e punho como indicadores no tratamento ortodôntico. Rev. Dental Press. Ortodon. Ortop. Facial, v.13, p.92-100, 2008.

NERY, M.F.; ESPÉCIE, M.A.; SIMÃO S.M. Marine tucuxi dolphin (Sotalia guianensis) injuries as a possible indicator of fisheries interaction in southeastern Brazil. Braz. J. Oceanography, v.56, p.313-316, 2008. 
OGDEN, J.A.; CONLOGUE, G.J.; RHODIN, A.G. Roentgenographic indicators of skeletal maturity in marine mammals (Cetacea). Skeletal Radiol., v.7, p.119-123, 1981.

PERRIN, W.F. Variation of spotted and spinner porpoise (genus Stenella) in the eastern tropical Pacific and Hawaii. Bull. Sripps Inst Oceanogr., v.21, p.1-206.1975.

RAMOS, R.M.A.; DI BENEDITO, A.P.M; LIMA, N.R.W. Growth parameters of Pontoporia blainvillei and Sotalia fluviatilis (Cetacea) in northern Rio de Janeiro, Brazil. Aquatic. Mamm., v.26, p.65-75, 2000.

ROSAS, F.C.W.; MONTEIRO-FILHO, E.L.A. Reproduction of the estuarine dolphin (Sotalia guianensis) of the coast of Paraná, southern Brazil. J. Mammol., v.83, p.507-515, 2002.

SANTOS, M.C.O.; ROSSO, S.; RAMOS, R.M.A. Age estimation of marine tucuxi dolphins (Sotalia fluviatilis) in southeastern Brazil. J. Mar. Biol. Assoc., v.83, p.233-236, 2003.
SCHIMIEGELOW, J.M.M. Estudos sobre cetáceos odontocetos encontrados em praias da região entre Iguape (SP) e

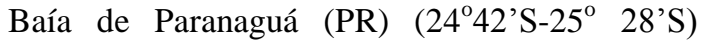
com especial referência a Sotalia fluviatilis (Gervais, 1853) (Delphinidae). 1990. 149f. Dissertação (Mestrado) - Instituto Oceanográfico, Universidade de São Paulo, São Paulo, SP.

SIMÕES-LOPES, P.C. Ocorrência de uma população de Sotalia fluviatilis (Gervais, 1853) (Cetacea, Delphinidae) no limite sul de sua distribuição, Santa Catarina, Brasil. Biotemas, v.1, p.57-62. 1988.

SISTEMAS de análises estatísticas - SAEG. Versão 9.1. Viçosa:UFV, 2007.

STOCKIN, K.A.; WISEMAN, N.; HARTMAN, A. et al. Use of radiography to determine age class and assist with the post-mortem diagnostics of a Bryde's whale (Balaenoptera brydei). N. Z. J. Mar. Freshwater Res., v.42, p.307-313, 2008. 